\title{
Shear wave elastography in varicocele patients: Prospective study to investigate correlation with semen parameters and histological findings
}

\author{
Andrea Fuschi $^{1,2}$ | Lorenzo Capone $^{1}$ | Saleh Abuorouq ${ }^{1}$ | Yazan Al Salhi ${ }^{1,2}$ | \\ Gennaro Velotti $^{1}$ | Sara Aversa ${ }^{3}$ | Flavia Carbone ${ }^{4}$ | Piero Maceroni ${ }^{2}$ | \\ Vincenzo Petrozza $^{3}$ | Antonio Carbone ${ }^{1,4}$ (D) | Antonio Luigi Pastore ${ }^{1,4}$ (D)
}

\author{
${ }^{1}$ Urology Unit, Department of Medico- \\ Surgical Sciences and Biotechnologies, \\ Faculty of Pharmacy and Medicine, Sapienza \\ University of Rome, Latina, Italy \\ ${ }^{2}$ ICOT - Surgery, Orthopedy, Traumatology \\ Institute, Latina, Italy \\ ${ }^{3}$ Histology Unit, Department of Medico- \\ Surgical Sciences and Biotechnologies, \\ Faculty of Pharmacy and Medicine, Sapienza \\ University of Rome, Latina, Italy \\ ${ }^{4}$ Uroresearch, Non-profit Association for \\ Research in Urology, Latina, Italy \\ Correspondence \\ Antonio Luigi Pastore, Urology Unit, \\ Department of Medical-Surgical Sciences \\ and Biotechnologies, Faculty of Pharmacy \\ and Medicine, "Sapienza" University of \\ Rome, Corso della Repubblica 79, 04100 \\ Latina (LT), Italy. \\ Email: antopast@hotmail.com
}

\begin{abstract}
Aims: Shear wave elastosonography (SWE) is a non-invasive ultrasound imaging modality used to assess the mechanical properties of tissues such as rigidity and elasticity. In this prospective study, we investigated the effect of laparoscopic varicocelectomy on the elasticity, degree of fibrosis and function of the testes through SWE and we evaluated the correlation with semen parameters and histology findings. Methods: Male patients with monolateral left varicocele and progressive alteration of the semen quality were enrolled prospectively. Patients were evaluated before varicocelectomy, 3 and 6 months after surgery with semen analysis, ecocolordoppler US and SWE. In all patients, a left testicular biopsy was performed the time of varicocelectomy and it was repeated after 6 months in 55\% of patients in order to investigate the histological findings and to correlate with SWE results.

Results: The study was conducted on 82 patients. SWE showed a statistically significant difference between left and right testicles. Three months after surgery the mean left testicular volume increased, mean left SWE features decreased, and sperm count increased ( $P$ values $<.0001$ ). The SWE parameters, testicular volume and semen analysis values showed a statistically significant positive correlation between the pre and postoperative results ( $P$ value $<.0001$ ). The histological alterations were significantly changed 6 months postoperative with a complete morphology recovery in accordance with SWE results.

Conclusions: SWE showed a statistically significant positive correlation with testicular volume, semen analysis and histological findings. This study represents the first investigation that correlates the varicocele, the testis volume, the quality of the seminal fluid ant the histological findings with the ultrasound and SWE values.
\end{abstract}

\section{1 | INTRODUCTION}

Shear wave elastosonography (SWE) is a non-invasive ultrasound (US) imaging modality used to assess the mechanical properties of tissues such as rigidity and elasticity. ${ }^{1}$ This technique allows the evaluation of the degree of fibrosis caused by parenchymal 
that can be used to differentiate benign and malignant testicular lesions. ${ }^{10}$ It can also be used for infertility assessment and as shown by Schurich et al, SWE can be used for structural analysis of testicular parenchyma and could become an additional method for detecting alterations in pathological tissues, such as those associated with varicocele. ${ }^{11}$ US with Doppler is currently the most widely used imaging technique for the assessment of varicocele and is usually performed in all patients to confirm the diagnosis. Male infertility can be caused by several factors and varicocele is the most frequent cause, with an estimated incidence of $35 \%-44 \%$ in patients with primary infertility and $45 \%-81 \%$ with secondary infertility. ${ }^{12}$ Lipshultz and Corriere demonstrated that varicoceles result in testicular atrophy in both fertile and sub-fertile men. ${ }^{13}$ Their findings suggested that varicoceles cause a progressive decline in fertility and can continue to induce impairment of spermatogenesis, despite prior fertility. SWE could evaluate the degree of testicular fibrosis, reflecting parenchymal damage and spermatogenesis impairment in patients with varicocele. In this prospective study, we investigated the effect of transperitoneal laparoscopic varicocelectomy on the elasticity, degree of fibrosis and function of testes by SWE. We further evaluated the findings of SWE to establish if there exists any correlation with semen parameters and histological findings.

\section{2 | MATERIALS AND METHODS}

Between October 2017 and December 2018, we prospectively enrolled male patients with a clinically significant monolateral left varicocele and a progressive alteration in semen quality according to World Health Organization (WHO) 2010 reference limits. ${ }^{14}$ The study was approved by the ethical committee of Sapienza University Pharmacy and Medicine Faculty, Latina, Italy. Written informed consent forms were obtained from all the patients before SWE and surgery. All study procedures involving human participants were performed in accordance with the ethical standards of the institution's national research committee and with the 1964 Helsinki Declaration and its later amendments or comparable ethical standards. Patients were evaluated with US, eco-colour Doppler examination and SWE; all these examinations were performed by the same radiologist having 21 years of experience in US and 8 years of experience in SWE. Patients were evaluated considering any concomitant comorbidities, drug assumption, objective examination (testicular size and consistency, varicocele degree), semen analysis and hormonal profile (LH, FSH and Total Testosterone). All imaging and clinical parameters were analysed before the surgical procedure and after 3 and 6 months of laparoscopic varicocelectomy. Exclusion criteria were previous varicocelectomy, bilateral varicocele, solitary testis, congenital testicular anomalies, azoospermia and patients with a history of radiation therapy or chemotherapy. After clinical evaluation of varicocele, all patients were studied with colour Doppler US and graded according to the Sarteschi Classification. ${ }^{15}$ Doppler US and sonoelastography quantitative imaging was performed in all the patients using "Supersonic Aixplorer" scanner with a $15 \mathrm{MHz}$ linear

\section{What's known}

- Shear wave elastosonography is a non-invasive ultrasound modality used to assess the mechanical properties of tissues such as rigidity and elasticity. As semi quantitative elastographic technique, SWE can be used for structural analysis of testicular parenchyma in patients affected by varicocele.

\section{What's new}

- This study represents the first works that correlates the varicocele, the testis volume, the quality of the seminal fluid ant the histological findings with the SWE parameters.

probe. All procedures were performed with patients in both supine and standing positions. Five measurements were performed on each testis: in the upper, anterior, middle, posterior and lower segments. The results were calculated in kilopascal $(\mathrm{kPa})$. The testes volume was calculated with the Lambert formula $(V=0.71 \times$ length $\times$ thickness $\times$ width) and expressed in millilitres $(\mathrm{mL}) .{ }^{16}$ The percentage of volume difference between both testes was determined using an established formula for calculating the testicular atrophy index: (contralateral testis volume - affected testis volume)/contralateral testis volume $\times 100$ and expressed as percentage. ${ }^{17}$

Semen samples were obtained for each patient twice, before and after 3 and 6 months of the surgery and the samples were analysed within 30 minutes of collection. The same laboratory performed all the analyses in order to avoid inter-laboratory variations. Semen volume, sperm count per millimetre (million/ $\mathrm{mL}$ ), total and progressive motility percent (\%), morphology and vitality percent (\%) were considered according to the WHO values. All the patients underwent transperitoneal laparoscopic ligation of the internal spermatic vein with testicular artery sparing, according to Ivanissevich technique by the same experienced surgeon. All the patients also underwent left testicular biopsy at the same time of varicocelectomy to investigate the histological features associated with varicocele and in order to correlate them with SWE results. At six months after surgery, a new biopsy of the left testicle was repeated in $55 \%$ of the patients to evaluate the modifications in histological features after varicocelectomy in accordance with SWE results.

\section{3 | STATISTICAL ANALYSIS}

Statistical analysis was performed using the Statistical Package for the Social Sciences (SPSS), version 25.0 (SPSS Inc, Chicago, IL). P values $<.05$ were considered statistically significant. $t$ test was used for the comparison between means of independent groups and 2-tailed t-test for paired samples with $95 \%$ confidence intervals was used for 
TABLE 1 Preoperative and postoperative results

\begin{tabular}{|c|c|c|c|c|}
\hline Parameters & Preoperative & At 3 months & At 6 months & $P$ value \\
\hline Age, mean years old (SD) & $27.44(6.09)$ & / & / & \\
\hline Grade of Varicocele, mean (SD) & $3.19(0.397)$ & / & / & \\
\hline Right testicular Volume, mL (SD) & $17.47(2.35)$ & $17.75(2.31)$ & $17.73(2.29)$ & .13 \\
\hline Left testicular Volume, $\mathrm{mL}$ (SD) & $12.83(2.27)$ & $17.26(2.75)$ & $17.29(2.58)$ & $<.0001$ \\
\hline$P$ value & $<.0001$ & .403 & .399 & \\
\hline SWE, mean $\mathrm{kPa}$ right (SD) & $2.075(0.39)$ & $2.063(0.39)$ & $2.068(0.41)$ & .43 \\
\hline SWE, mean kPa left (SD) & $2.56(0.814)$ & $1.79(0.488)$ & $1.75(0.464)$ & $<.0001$ \\
\hline$P$ value & .002 & .009 & .007 & \\
\hline Count of sperm, mean $\mathrm{mL} / \mathrm{mil}$ (SD) & $24.17(17.54)$ & $38.06(23.02)$ & $40.81(23.01)$ & $<.0001$ \\
\hline $\begin{array}{l}\text { Total sperm motility at } 2 \text { hours, } \\
\text { mean \% (SD) }\end{array}$ & $31.88(20.7)$ & 37.19 (15.13) & $39.09(13.71)$ & .07 \\
\hline Normal sperm forms, mean \% (SD) & $32.25(24.46)$ & $26.81(18.08)$ & $26.78(17.93)$ & .145 \\
\hline
\end{tabular}

Abbreviations: kPa, kiloPascal; SD, standard deviation; SWE, shear wave elastography. comparison of continuous variables between dependent samples. The relation between the stiffness and the considered parameters was studied using linear regression and Pearson's correlation test.

\section{4 | RESULTS}

This study enrolled 82 male patients, according to inclusion and exclusion criteria, with a mean age of 27.44 years (SD: 6.095). Preoperative Doppler US showed a clinically significant left varicocele in all patients (Grade 3:81\% and Grade 4:19\%; SD: 0.397) without any significant hormonal value alterations. SWE and US volume of the right testis did not report any significant difference between preoperative and postoperative evaluation ( $P$ values $>.05$ ). As shown in Table 1, a statistically significant difference was observed in comparing baseline SWE results and the US volume of left and right testicles ( $P$ values: .002 and $<.0001$, respectively). Furthermore, the comparisons of baseline and postoperative SWE left testicle parameters were statistically significant ( $P$ value $<.0001)$.

At first postoperative evaluation after 3 months from surgery, the mean left testicular volume increased from $12.83 \mathrm{~mL}$ to $17.26 \mathrm{~mL}$ (SD: $1.85 ; P$ value $<.0001)$ and no significant difference was reported regarding the postoperative testicular volume of both the testes ( $P$ value: .403). Mean left SWE decreased from 2.56 to $1.79 \mathrm{kPa}$ (SD: 0.38; $P$ value <.0001) and sperm count increased from $24.17 \mathrm{mil} / \mathrm{mL}$ to 38.06 $\mathrm{mil} / \mathrm{mL}$ (SD: $12.40 ; P$ value $<.0001$ ). There was no statistically significant increase in total sperm motility at 2 hours which increased from 31.88 seconds to 37.19 seconds (SD: 16.01 ; $P$ value: .07, Table 1 ).

The correlation between SWE parameters, testicular volume and semen analysis values showed a statistically significant positive correlation between the difference of pre and postoperative left SWE and testicular volume $(r:+0.611 ; P$ value $<.0001)$. This positive correlation was found to be associated with a significant negative correlation between postoperative SWE of left testis and ipsilateral testicular volume and sperm count at 3 months $(r$ : -0.529 and -0.422 , respectively; $P$ value $<.0001$ )
At 6 months, minor improvements in the analysed parameters were reported, but when compared with the results at 3 months, these improvements did not show any statistical difference. No significant differences were shown at 3 and 6 months in the percentage of normal sperm forms. All patients were discharged the next day without any postoperative complications. At 6 months, changes in serum hormonal levels (FSH, LH, Testosterone) and varicocele recurrences were recorded, if any.

Histological findings of the testis biopsies (performed during varicocelectomy) reported the thickening of the germinal epithelium with increased apoptosis, Sertoli and germ cells cytoplasmic vacuolisation, and alterations in spermatids and spermatozoa. In all evaluated cases ( 45 out of 82 pts), the 6-months postoperative testicular biopsies revealed a complete morphological recovery with significant disappearance of epithelium thickening, apoptosis and vacuolisation. These postoperative data were in accordance with SWE results.

\section{5 | DISCUSSION}

Varicocele contributes to both primary and secondary male infertility. The estimated varicocele incidences are about $40 \%$ in male patients as per published reports. ${ }^{1}$ This pathological condition has a higher probability to affect the left testicle than the right side $(80 \%$ $90 \%$ and $1 \%-7 \%$, respectively). ${ }^{18,19}$ US is currently the most widely used imaging technique for the assessment of varicocele, but its role in the diagnostic algorithm is still controversial. There is no universal and recognised system to classify varicocele severity because of an insufficient correlation between ultrasonographic classification system and clinical assessment of varicocele severity. SWE might represent a diagnostic tool capable of supporting clinical diagnosis by providing a qualitative and quantitative assessment of testicular stiffness. SWE is a widespread imaging tool used in the assessment of liver fibrosis and evaluation of breast, thyroid, prostate and testes nodules. However, it was previously never used to assess testicular 


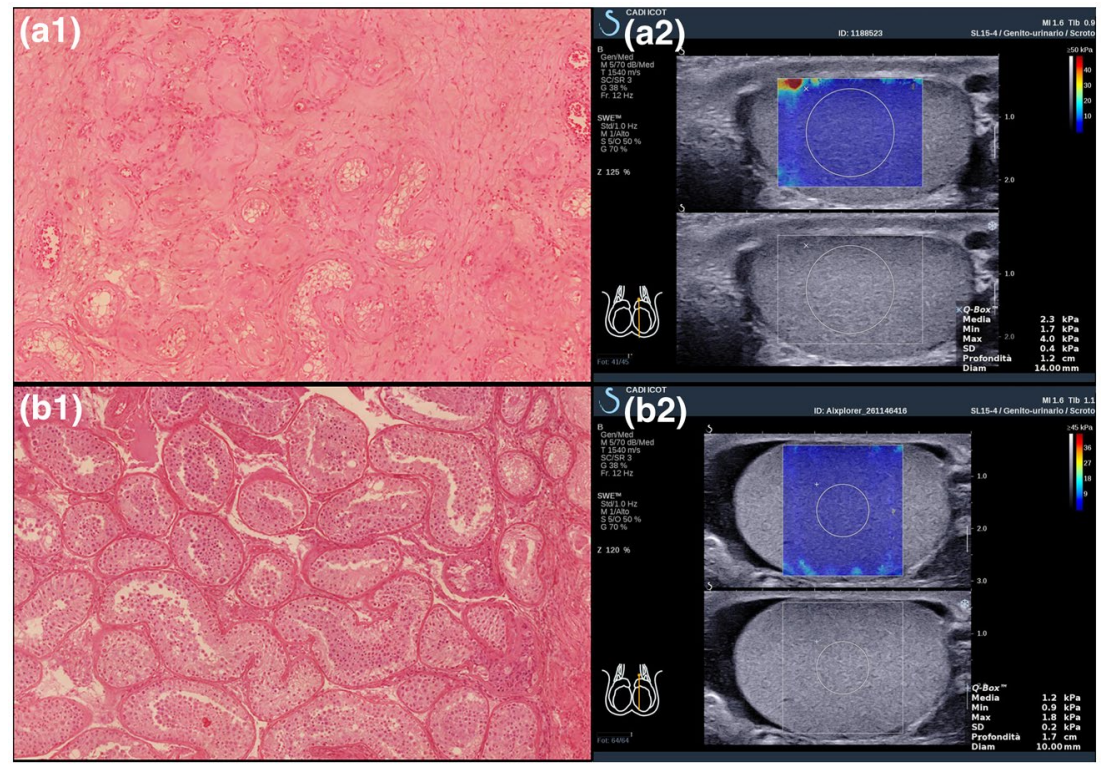

FIGURE 1 (a1) Histological findings at the time of varicocelectomy: thickening of germinal epithelium, Sertoli and germ cells cytoplasmic vacuolisation and alterations in spermatids and spermatozoa. (a2) Testicular SWE before surgery: the measurement was performed in the central region of testicular parenchyma to avoid high stiffness of peripheral portions (albuginea) and septa. A high-frequency linear probe (15-4 MHz) with a stiffness scale expressed in $\mathrm{kPa}$ (range: 10-50 kPa) was used. The measurement was performed in the absence of compression to avoid increased stiffness. (b1) Histology 6 months after varicocelectomy: complete normal testicular morphology recovery. (b2) Testicular SWE after surgery: the area of interest (ROI) to measure the stiffness was centrally positioned with the indication of the max value $(1.8 \mathrm{kPa})$, the min value $(0.9 \mathrm{kPa})$ and the mean value $(1.2 \mathrm{kPa})$. The standard deviation of all measurements including ROI was also calculated in order to determine the reliability of measurements

stiffness with respect to the issue of fertility. ${ }^{20-24}$ Recently, the diagnostic role of SWE in detecting different elasticity values depends on testicular volume and functional properties as previously demonstrated. ${ }^{25}$ In this study, we correlated the pre and postoperative SWE features with histopathological findings (Figure 1). Our study finds a strong correlation between testicular fibrosis and SWE data in pre and postoperative evaluation.

The basic principle of SWE is based on the application of US probes to produce an acoustic push pulse for generating shear waves from which tissue stiffness can be determined. The underlying mechanism is that the acceleration of propagating shear waves through harder tissue (stiffer testes) will be faster and slower through the softer tissues (normal testes). ${ }^{3,26}$ Erdogan et al found that SWE can be used as an effective technique to assess testicular stiffness in patients with varicocele and predict interstitial fibrosis and the severity of histological damage. However, findings were not directly correlated to testicular volume and the degree of parenchymal damage. ${ }^{27}$ In our study, we found that subjects with left varicocele have a greater testicular stiffness than normal subjects; this data was confirmed when the left testicle was compared with the contralateral one. At 3 months after the surgical procedure, a decrease in stiffness and an increase in the volume of testicle previously affected by varicocele were observed in all patients ( $P$ value $<.0001$ ), suggesting a significant correlation with the volume of the testicle.

In the study by Abdelwahab and colleagues, preoperative testicular elastography results were found to be a possible predictor of semen analysis changes before and after varicocelectomy. ${ }^{28}$ Authors showed a statistically significant negative correlation between the SWE stiffness index and both sperm count and total motility. This data on semen parameters and testicular SWE is in accordance with the results of our study. Moreover, our findings further added the correlation between the testicular volume evaluation and SWE data to these investigated parameters.

The preoperative mean left testicular volume was $12.83 \mathrm{~mL}$ and mean SWE was $2.56 \mathrm{kPa}$, and three months after surgery, the left testicular volume was $17.26 \mathrm{~mL}$ and SWE was $1.79 \mathrm{kPa}$. The results 6 months after surgery remain substantially unchanged (volume $17.29 \mathrm{~mL}$; SWE 1.75 kPa) (Table 1). Various studies have revealed that testicular volume has a significant association with semen volume, sperm count and motility. ${ }^{29,30}$ In their preliminary study, Condorelli et al reported that sperm parameters worsen with decreasing testicular volume. ${ }^{31}$ Thus, the testicular size could be a reliable parameter that might reflect the degree of parenchymal condition, but further studies with larger samples of patients are needed to confirm this finding.

Additionally, Kucukdurmaz et al stated that there is a significant correlation between testis volume and sperm counts in patients with normal semen fluid analysis. ${ }^{32}$ However, they failed to demonstrate this correlation in patients with abnormal semen fluid analysis. Furthermore, they showed no relation between testicular size and total motile sperm, which is concordant to the findings of our study that did not show a significant increase in motility sperm rate (from $31.88 \%$ at baseline to $37.19 \%$ and $39.09 \%$ at three and six months after surgery, respectively; $P$ value $>.05$ ) and normal 
sperm morphology rates. The other seminal parameter, morphology, although not statistically significant, showed a paradossale decline rather than an increase from $32.25 \%$ at baseline to $26.81 \%$ and $26.78 \%$ at three and six months after surgery, respectively. These results could be because of the limited sample size of analysed patients.

As expected, in all the patients, a postoperative pronounced increase in sperm count was found with $24.17 \mathrm{mil} / \mathrm{mL}, 38.06 \mathrm{mil} / \mathrm{mL}$ and $40.81 \mathrm{mil} / \mathrm{mL}$ at baseline, three months and six months postoperative, respectively.

Dede et al investigated the relationship between elastography scores, serum FSH levels and varicocele. ${ }^{1}$ They found an inverse correlation of testicular elasticity with a grade of varicocele and serum FSH values. Our study did not show any correlation between the improvement in stiffness of the testes and change in serum hormonal level pre and postoperatively (FSH, LH and testosterone). Moreover, Kucukdurmaz et al found no association between varicocele and tissue elasticity and between FSH levels and elastography findings amongst their patient groups. ${ }^{32}$ This study has several main limitations. The sample size and limited follow-up term might influence the interesting and encouraging results obtained in this study on a novel non-invasive diagnostic method strongly requested to investigate fertility and varicocele. To the best of our knowledge, this is the first study that correlates varicocele, testis volume, quality of the seminal fluid and histological findings with the US and SWE values.

\section{6 | CONCLUSION}

Testicular SWE is a feasible and reproducible technique that can be used in the assessment of male infertility with clinically detectable varicocele to provide additional quantitative information. Our study demonstrated a significant difference in terms of stiffness, testicular volume and quality of spermatic liquid in patients undergoing laparoscopic transperitoneal varicocelectomy.

\section{DISCLOSURES}

The authors have no conflict of interest to disclose.

\section{AUTHORS' CONTRIBUTIONS}

Andrea Fuschi, Yazan Al Salhi, Antonio Luigi Pastore designed the study and wrote the manuscript. Flavia Carbone, Piero Maceroni, Sara Aversa, Alessia Martoccia contributed to experimental design and writing, Yazan Al Salhi, Flavia Carbone, Vincenzo Petrozza, Antonio Carbone provided patient material and contributed to the experimental design of the study. Andrea Fuschi performed the statistical evaluation. Gennaro Velotti, Saleh Abouruq, Sara Aversa, Lorenzo Capone contributed to the experimental design and approved for the final version and submission. All authors have read and approved the final version of the manuscript.

\section{DATA AVAILABILITY STATEMENT}

The datasets used and/or analysed during the current study are available from the corresponding author on reasonable request.

\section{ETHICS APPROVAL AND CONSENT TO PARTICIPATE}

This study was approved by the Medical Ethics Committee of Sapienza University Pharmacy and Medicine Faculty, Latina, Italy (LT n. 2017/ANDRURO UNIV 49699) and written informed consent was obtained from all the patients.

\section{PATIENT CONSENT FOR PUBLICATION}

All patients give their consent for publication.

\section{ORCID}

Antonio Carbone iD https://orcid.org/0000-0003-1725-1933

Antonio Luigi Pastore iD https://orcid.org/0000-0003-4293-9174

\section{REFERENCES}

1. Dede O, Teke M, Daggulli M, et al. Elastography to assess the effect of varicoceles on testes: a prospective controlled study. Andrologia. 2016;48:257e61.

2. Magri F, Chytiris S, Capelli V, et al. Shear wave elastography in the diagnosis of thyroid nodules: feasibility in the case of coexistence of chronic autoimmune Hashimoto's thyroiditis. Clin Endocrinol (Oxf). 2012;76(1):137-141. https://doi. org/10.1111/j.1365-2265.2011.04170.x

3. Cosgrove DO, Berg WA, Dore CJ, et al. Shear wave elastography for breast masses is highly reproducible. Eur Radiol. 2012;22:1023-1032.

4. Ozturker C, Karagoz E, Incedayi M. Non-invasive evaluation of liver fibrosis: 2-D shear wave elastography, transient elastography or acoustic radiation force impulse imaging? Ultrasound Med Biol. 2016;42:3052

5. Hekimoglu K, Yildirim Donmez F, Arslan S, et al. The role of shear wave elastography in the diagnosis of chronic autoimmune thyroiditis. Med Ultrason. 2015;17:322-326.

6. Guibal A, Boularan C, Bruce M, et al. Evaluation of shear wave elastography for characterization of focal liver lesions on ultrasound. Eur Radiol. 2013;23:1138-1149.

7. Rafaelsen SR, Vagn-Hansen C, Sorensen T, et al. Ultrasound elastography in patients with rectal cancer treated with chemoradiation. Eur J Radiol. 2013;82:913-917.

8. Hamidi C, Goya C, Hattapoglu S, et al. Acoustic radiation force impulse (ARFI)imaging for the distinction between benign and malignant thyroid nodules. Radio Med. 2015;120:579-583.

9. Onur MR, Poyraz AK, Bozgeyik Z, et al. Utility of semiquantitative strain elastography for differentiation between benign and malignant solid renal masses. J Ultrasound Med. 2015;34:639-647.

10. Onur MR, Poyraz AK, Ucak EE, et al. Semi-quantitative strain elastography of liver masses. J Ultrasound Med. 2012;31:1061-1067.

11. Schurich $M$, Aigner F, Frauscher $F$, et al. The role of ultrasound in assessment of male fertility. Eur J Obstet Gynecol Reprod Biol. 2009;144:S192-S198.

12. Damsgaard J, Joensen UN, Carlsen E, et al. Varicocele is associated with impaired semen quality and reproductive hormone levels: a study of 7035 healthy young men from six European countries. Eur Urol. 70:1019-1029.

13. Lipshultz LI, Corriere JN Jr. Progressive testicular atrophy in the varicocele patient. J Urol. 1977;117:175-176.

14. Cooper TG, Noonan E, von Eckardstein S, et al. World Health Organization reference values for human semen characteristics. Hum Reprod Update. 2009;16:231-245.

15. Sarteschi LM. Lo studio del varicocele con color Doppler. G Ital Ultrasonologia. 1993;4:43-49

16. Lambert B. The frequency of mumps and mumps orchitis, and the consequences for sexuality and fertility. Acta Genet Suppl. 1951;2:1. 
17. Niedzielski J, Pisarka K, Przwratil P. The usefulness of testicular atrophy index in the assessment of undescended testicle-preliminary report. Ann Acad Med Stetin. 2003;48:112-114.

18. Scmiz I, Tokgoz O, Tokgoz H, et al. The investigation of correlation between semen analysis parameters and intraparenchymal testicular spectral Doppler indices in patients with clinical varicocele. Ultrasound Q. 2014;30:33-40.

19. Kass EJ, Stork BR, Steinert BW. Varicocele in adolescence induces left and right testicular volume loss. BJU Int. 2001;87:499-501.

20. Zhang X, Lv F, Tang J. Shear wave elastography (SWE) is reliable method for testicular spermatogenesis evaluation after torsion. Int J Clin Exp Med. 2015;8:7089-7097.

21. Aigner F, De Zordo T, Pallwein-Prettner L, et al. Real-time sonoelastography for the evaluation of testicular lesions. Radiology. 2012;263:584-589.

22. Daniaux M, Auer T, De Zordo T, et al. Strain elastography of breast and prostate cancer: similarities and differences. Rofo. 2016;188:253-258.

23. Dyrla P, Gil J, Florek M, et al. Elastography in pancreatic solid tumours diagnosis. Prz Gastroenterol. 2015;10:41-46.

24. Lu Q, Ling W, Lu C, et al. Hepatocellular carcinoma: stiffness value and ratio to discriminate malignant from benign focal liver lesions. Radiology. 2015;275:880-888.

25. De Zordo T, Stronegger D, Pallwein-Prettner L, et al. Multiparametric ultrasonography of the testicles. Nat Rev Urol. 2013;10: 135-148.

26. Sigrist RMS, Liau J, Kaffas AE, et al. Ultrasound elastography: review of techniques and clinical applications. Theranostics. 2017;7:1303-1329.
27. Erdogan H, Durmaz MS, Arsalan S, et al. Shear wave elastography evaluation of testes in patients with varicocele. Ultrasound Q. 2020 Mar;36:64-68.

28. Abdelwahab K, Eliwa AM, Seleem MM, et al. Role of preoperative testicular shear wave elastography in predicting improvement of semen parameters after varicocelectomy for male patients with primary infertility. Urology. 2017;107:103-106. https://doi.org/10.1016/j.urolo gy.2017.04.026

29. Kumar S, Mohsen N, Vineeth VS, et al. Assessment of testicular volume in correlation with spermiogram of infertile males in South India. Adv Stud Biol. 2013;5:327-335.

30. Kristo A, Dani E. The correlation between ultrasound testicular volume and conventional semen parameters in Albanian subfertile males. Maced J Med Sci. 2014;7:464-466.

31. Condorelli R, Calogero AE, La Vignera S. Relationship between testicular volume and conventional or nonconventional sperm parameters. Int J Endocrinol. 2013;2013:145792.

32. Küçükdurmaz F, Sarıca MA, Emre Ö, et al. Evaluation of the diagnostic efficacy of strain elastography in infertile population with normal and abnormal semen parameters. Turk J Urol. 2017;43:261-267.

How to cite this article: Fuschi A, Capone L, Abuorouq S, et al. Shear wave elastography in varicocele patients: Prospective study to investigate correlation with semen parameters and histological findings. Int J Clin Pract. 2020;00:e13699. https://doi.org/10.1111/ijcp.13699 